\title{
Dos métodos para la cuantificación de paraquat en plaguicidas tipo Concentrado Soluble
}

\author{
Ericka Barrantes Barrantes $^{1}$ iD) Esteban Pérez López ${ }^{1}$ (D) Roberto Villalobos Landazuri $^{3}$ (D) \\ 1. Universidad de Costa Rica, Sede de Occidente, 20305 Grecia, Costa Rica; erickabb97@gmail.com; \\ esteban.perezlopez@ucr.ac.cr \\ 2. Servicio Fitosanitario del Estado, 10108 San José, Costa Rica; rvillalobos@sfe.go.cr
}

Recibido 13-VII-2020 • Corregido 21-IX-2020 • Aceptado 26-X-2020

DOI: https://doi.org/10.22458/urj.v12i2.3042

\begin{abstract}
Two methods for the quantification of paraquat in Soluble Concentrate pesticides". Introduction: In Costa Rica, paraquat is the third most imported pesticide and it is widely applied in different plantations. This makes it necessary to have a strict quality control for compliance with local regulations Objective: To verify and compare AOAC standard methods 969.09 by UV-Vis spectrometry and the respective CIPAC method by HPLC. Method: The guidelines described by the Costa Rican Accreditation Body (ECA) were followed to verify analytical performance parameters in standardized methods. Results: The UV and HPLC methods proved suitable but had significant differences in the results. Conclusions: Both methodologies comply with the requirements but we recommend an enlarged test with more analysts and laboratories to define which is more accurate.
\end{abstract}

Keywords: Pesticides, paraquat, methodology, verification, quality control.
RESUMEN. Introducción: En Costa Rica, el paraquat es el tercer plaguicida más importado y se aplica ampliamente en diferentes cultivos. Esto hace necesario un estricto control de calidad para el cumplimiento de la normativa local. Objetivo: Verificar y comparar los métodos estándar AOAC 969.09 por espectrometría UV-Vis y el respectivo método CIPAC por HPLC. Método: Se siguieron los lineamientos descritos por el Organismo de Acreditación de Costa Rica (ECA) para verificar los parámetros de desempeño analítico en métodos estandarizados. Resultados: Los métodos UV y HPLC demostraron ser adecuados, pero tuvieron diferencias significativas en sus resultados. Conclusiones: Ambas metodologías cumplen con los requisitos, pero recomendamos una prueba ampliada con más analistas y laboratorios para definir cuál es más precisa.

Palabras clave: Plaguicidas, paraquat, metodología, verificación, control de calidad.

La intensificación de la agricultura motivada por la necesidad de proveer productos agrícolas a una población cada día creciente, trae como consecuencia la proliferación de plagas y enfermedades. Las plagas se encuentran entre los factores limitantes más importantes de la productividad de los sistemas agroforestales y pecuarios, sean insectos, patógenos o malezas estos organismos son responsables del $37 \%$ al $50 \%$ de las pérdidas reportadas en la agricultura mundial (Echemendía, s.f.).

Por ello, es fundamental que los productores realicen un manejo integrado de plagas, siendo los plaguicidas una herramienta de gran importancia debido a su efectividad a corto plazo y el cumplimiento de las exigencias del mercado. Sin embargo, estos productos tienen efectos secundarios nocivos como la contaminación de las aguas superficiales y subterráneas durante los procesos de fabricación o aplicación de los mismos. También, se ha demostrado en el ser humano, que la exposición a plaguicidas puede reducir la producción y función de los linfocitos o glóbulos blancos y otras células del sistema defensivo, incrementando el riesgo de enfermedades infecciosas, parasíticas y de cánceres asociados con inmunosupresión (Echemendía, s.f.).

La agroquímica es la rama de la ciencia que estudia la producción agrícola, la transformación de productos crudos en alimentos y bebidas, y la vigilancia del medio ambiente y la remediación. Además, se dice que estudia las relaciones recíprocas entre las plantas, suelos, fertilizantes y 
plaguicidas durante el proceso del cultivo de las plantaciones agrícolas, el ciclo de nutrientes en la agricultura y la aplicación de fertilizantes y plaguicidas con el propósito de incrementar la cosecha, mejorar su calidad, aumentar la fertilidad del suelo y disminuir el ataque de organismo no deseados (Reyes, Vásquez, \& González, 2010).

Los plaguicidas son compuestos orgánicos e inorgánicos que se emplean para luchar contra los parásitos de los cultivos, clasificados en: insecticidas, herbicidas, acaricidas, fungicidas y rodenticidas. El ingrediente activo de la mayoría de plaguicidas se encuentra comprendido en un rango entre $75-98 \%$, siendo el resto productos secundarios e impurezas de fabricación. Presentan características típicas tales como: toxicidad, elevada resistencia a la degradación y carácter acumulativo (Marín, 2003).

Al aplicar cualquier plaguicida se inicia un proceso de interacción entre éste y el medio hasta que termina su efecto y desaparece. Esta interacción comprende la atmósfera, suelo, agua y ecosistemas. Algunos plaguicidas según su estructura y características fisicoquímicas persisten en el ambiente, propiciando con ello la acumulación en agua y suelo principalmente, subiendo después por la cadena trófica y llegando hasta los seres humanos (Salazar \& Aldana, 2011).

Los plaguicidas se dividen en varios subtipos que son utilizados según la necesidad que se desee cubrir. Específicamente los herbicidas, son sustancias o mezclas empleadas para eliminar plantas o malas hierbas que son consideradas indeseables y que crecen constantemente en diversas partes del suelo y que pueden afectar una plantación de agricultura o bien por estética de un determinado lugar (Mendoza, 2011).

El paraquat es uno de los herbicidas más utilizados para controlar maleza en muchos sitios agrícolas y de uso no-agrícola. Incluso es empleado como un defoliante en cultivos como el algodón con anterioridad a la cosecha (Agencia de Protección Ambiental de Estado Unidos, 2019). También, es fundamental saber que el paraquat se considera moderadamente tóxico debido a que una exposición muy corta podría causar la muerte o lesiones residuales importantes, aunque se proporcione un rápido tratamiento médico a la persona, además es altamente persistente ya que puede durar hasta 3 años en el ambiente (Inquisa, s.f.). Es un plaguicida de tipo SL, los cuales son líquidos homogéneos que al ser diluidos directamente en agua, forman una solución verdadera del activo, pudiendo contener auxiliares de formulación insolubles. Se obtienen a partir de un concentrado soluble que se diluye en agua. (Agrologica, 2012).

En México se estudió la degradación fotocatalítica de soluciones acuosas de paraquat con dióxido de titanio iluminado con luz ultravioleta $(365 \mathrm{~nm})$, donde se comprobó que es posible transformar a la molécula de paraquat en otras moléculas orgánicas de estructura más sencilla que a su vez se transforman a dióxido de carbono. Se analizó la concentración de paraquat por medio de la espectrometría UV-Vis (Monctezuma, Zamarripa, \& Leyva, 2003).

También, en Argentina se creó el método de análisis por inyección en flujo y detección espectrofotométrica para la determinación cuantitativa de paraquat en muestras de orina, con la formación de un producto coloreado $(600 \mathrm{~nm})$ posterior a la reducción de paraquat con glucosa en un medio alcalino mediante un sistema en línea. Se determinó que bajo las condiciones óptimas de

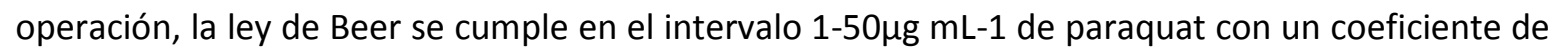
correlación $>0,999$, además el estudio de recuperación osciló entre 97,9 y 102,1\%. Por último, el método analítico fue aplicado satisfactoriamente al análisis de muestras de dos pacientes intoxicados con paraquat (Luna, Di Bernardo, García, Ovalles, \& Calderón, 2008).

Además, en Brasil se realizó una investigación sobre la degradación del paraquat a través de la ozonización en agua. Se determinó la influencia de la concentración del ozono disuelto, pH, flujo volumétrico de oxígeno y la velocidad rotacional del agitador en la eficiencia del método; donde se obtuvo la mejor eficiencia cuando se trabajó a un pH 3,7,100rpm de agitación y un flujo de 9L/h. La determinación de la concentración del paraquat se hizo a través del método de espectrofotómetro 
de absorción y se demostró que aunque el ozono es rápido y eficiente para eliminar el paraquat, se tiene que realizar un análisis cuidadoso de los productos de degradación para evaluar la ganancia total del proceso en relación con la degradación de los compuestos originales y la formación de productos potencialmente dañinos (Reynoso \& Carvalho, 2010).

Por otro lado, en Cuba se realizó una investigación para determinar plaguicidas organofosforados y organoclorados en aguas superficiales, suelos y sedimentos; analizándose también los PCB, mediante los métodos analíticos recomendados por el Marine Environmental Study Laboratory de la IAEAE. Se escogió Zapata debido a que destacan campos arroceros, áreas citrícolas y cultivos de caña de azúcar; aunque el de mayor riesgo ambiental es el arroz por la cantidad y forma (por lo general el avión) en que se aplican los plaguicidas. Los resultados de los análisis demostraron que no hubo presencia de casi ningún plaguicida en las muestras, incluido el paraquat, excepto por el DDT (Dierksmeier et al., 2002).

Asimismo, en Venezuela, específicamente en la comunidad agrícola de Bailadores, se monitorearon los ríos Las Tapias, Las Playitas y Mocoties por la presencia de residuos de plaguicidas durante 2008 y 2010. Se analizó la presencia de residuos de plaguicidas mediante HPLC con detector de arreglo de diodos, con el cual se obtuvo como resultado clorpirifos $(302,9 \pm 0,9 \mu \mathrm{g} / \mathrm{L})$, diazinon $(459 \pm 4,0 \mu \mathrm{g} / \mathrm{L})$, dimetoato $(55 \pm 3,0 \mu \mathrm{g} / \mathrm{L})$, mancozeb $(108 \pm 1,0 \mu \mathrm{g} / \mathrm{L})$ y metamidofos $(107 \pm 8,0 \mu \mathrm{g} / \mathrm{L})$; pero no se encontró residuo de Paraquat en las aguas superficiales (Molina, Flores, Balza, Benítez, \& Miranda, 2012).

Por su parte, en Colombia se validó el método de voltametría de onda cuadrada para la cuantificación de paraquat, siendo los parámetros evaluados el límite de detección, límite de cuantificación, sensibilidad, precisión y exactitud. Luego se analizaron diez muestras de aguas superficiales por triplicado, recolectadas en forma aleatoria en las veredas de Puente Boyacá, Bojirque y Montoya; donde dicho territorio está dedicado a labores agrícolas, dentro de las cuales se destacan los cultivos de papa y zanahoria. La concentración de paraquat fue entre 0,011 y $1,572 \mathrm{mg} / \mathrm{L}$, los cuales evidencian la presencia de este herbicida en los tres ríos evaluados, siendo los valores obtenidos comparados con los resultados de contramuestras analizadas por la técnica de espectroscopia UV-Vis según el método 969,09 de la AOAC (Alza-Camacho, García-Colmenares, \& Chaparro-Acuña, 2016).

En el caso de Costa Rica, éste es de los mayores consumidores de plaguicidas químicos en la agricultura a nivel mundial (Araya, 2015), y se han realizado determinaciones por medio de la técnica HPLC en aguas superficiales, específicamente en la cuenca del Río San Blas, donde no se encontraron residuos de paraquat pero si de otros plaguicidas (Morales, 2013).

Por último, en el Laboratorio de Control de Calidad de Agroquímicos de Costa Rica se realizó la determinación de terpiridinas totales en materia prima de Dicloruro de Paraquat por medio de cromatografía de gases acoplado a masas. Se logró validar el método y determinar la linealidad, rango, límite de detección y cuantificación, repetibilidad, veracidad y robustez. Además, se midieron once muestras diferentes de paraquat, donde cinco muestras presentaron valores promedio entre 0,3 y $0,5 \mathrm{mg} / \mathrm{L}$, otras tres muestras obtuvieron valores promedio de 0,7 y $1 \mathrm{mg} / \mathrm{L}$, y las tres muestras restantes sobrepasaron el límite de la normativa de la Organización de las Naciones Unidas para la Alimentación y la Agricultura (FAO por sus siglas en inglés), siendo este $1 \mathrm{mg} / \mathrm{L}$, con valores promedio entre 1,1 y $1,35 \mathrm{mg} / \mathrm{L}$. (Delgado, 2015). 


\section{MATERIALES Y MÉTODOS}

Se verificaron los parámetros de desempeño analíticos para métodos normalizados dictados por el ECA en el documento "ECA-MC-PO01-G01 Guía de Validación V03". Los métodos normalizados para determinación de paraquat, contemplados en este estudio, son: el 969,09 de la AOAC por espectrometría UV-Vis y el método respectivo del CIPAC por HPLC. La verificación, tanto experimental como estadística, de cada uno de los parámetros de desempeño analítico se evaluó de la siguiente manera:

Selectividad-especificidad: Se determina al medir un blanco por triplicado y comparar el espectro o cromatograma obtenido con el de los patrones y muestras, empleados en linealidad y repetibilidad respectivamente, para asegurar que la señal adquirida se debe solamente al analito que se desea cuantificar y no a interferencias.

El blanco empleado al utilizar el método de la AOAC por espectrometría UV-Vis, se prepara al agregar $10 \mathrm{~mL}$ de la solución de ditionito de sodio al $1 \% \mathrm{~m} / \mathrm{m}$ en un balón de $100 \mathrm{~mL}$ y llevar a volumen con agua desionizada. En cambio, el blanco utilizado por el método de CIPAC se prepara al añadir $10 \mathrm{~mL}$ de acetronitrilo en un balón de $100 \mathrm{~mL}$, se lleva a la marca de aforo con agua desionizada, se filtra e introduce en el vial para hacer la medición a $290 \mathrm{~nm}$.

Es importante explicar, que la solución de ditionito de sodio $1 \% \mathrm{~m} / \mathrm{m}$ se alista al pesar $1 \mathrm{~g}$ de ditionito de sodio, disolverlo y llevar a la marca de aforo, en un balón de $100 \mathrm{~mL}$ de color ámbar, con hidróxido de sodio al 0,1N.

Linealidad: Se construye una curva de calibración de cuatro patrones de $\pm 20 \%$ del valor en que se espera que se encuentre las muestras comerciales que se analizan cotidianamente. Se realiza solamente una curva y cuatro patrones debido a que es una verificación de la linealidad, por lo que no es necesario ejecutarla más veces o con mayor cantidad de patrones, además así se encuentra establecida en el método de la AOAC.

Para el método de la $A O A C$, se efectúa al seguir el siguiente procedimiento: Primero se prepara la Solución A: se seca el estándar de paraquat hasta llevarse a peso constante entre 100 y $120^{\circ} \mathrm{C}$. Luego se pesa $0,1728 \mathrm{~g}$ de dicloruro de paraquat $(72,4 \%$ del catión), se disuelve en agua desionizada y se diluye a $500 \mathrm{~mL}$ ( $1 \mathrm{~mL}$ contiene $0,25 \mathrm{mg}$ de paraquat).

Posteriormente se alista la Solución B: se pipetea $50 \mathrm{~mL}$ de la solución A en un balón de $250 \mathrm{~mL}$ y se lleva a volumen con agua desionizada.

Después, se agrega alícuotas de la solución B de $5,10,15$ y $20 \mathrm{~mL}$ en balones de $100 \mathrm{~mL}$. Al momento de adicionar los $10 \mathrm{~mL}$ de ditionito de sodio se debe llevar a volumen y medir inmediatamente a $600 \mathrm{~nm}$ porque el color se degrada con la luz, por lo que los patrones se miden de uno en uno.

Por otro lado, para el método de CIPAC se realiza de la siguiente forma: Solución Madre: se pesa $121,6 \mathrm{mg}$ de ion paraquat del estándar en un balón aforado de $100 \mathrm{~mL}$, se disuelve con agua desionizada, se coloca por 5 minutos en el baño ultrasónico y se lleva a volumen.

Es importante mencionar, que este método no posee curva de calibración ya que se emplea mediante la comparación de un estándar, el cual se prepara al tomar una alícuota de $10 \mathrm{~mL}$ de la solución madre y acetonitrilo llevándolo a volumen con agua desionizada en un balón de $100 \mathrm{~mL}$. Por lo que, se prepararon tres patrones adicionales de la siguiente manera: se tomaron alícuotas de $4,4,5$ y $6 \mathrm{~mL}$ de la madre, se agregaron $5 \mathrm{~mL}$ de acetronitrilo, se diluyó hasta $50 \mathrm{~mL}$ en un balón aforado con agua desionizada, se filtra e introduce en los viales para hacer la medición a 290nm. Por último, el r cuadrado de la curva de calibración no debe ser menor a 0,99.

Repetibilidad y precisión intermedia: La preparación de la muestra por el método de la AOAC es la siguiente: En un balón de $500 \mathrm{~mL}$, se pesa $0,25 \mathrm{~g}$ del ión paraquat y se lleva a volumen 
con agua desionizada (solución I). Luego, se toma una alícuota de $10 \mathrm{~mL}$ de la solución I y se diluye a $100 \mathrm{~mL}$ en un balón aforado (solución II). Por último, se pipetean $10 \mathrm{~mL}$ de la solución II y ditionito de sodio en un balón de $100 \mathrm{~mL}$, se lleva hasta la marca de aforo con agua desionizada y se mide instantáneamente a $600 \mathrm{~nm}$.

Respecto al método de CIPAC, las muestras se preparan al seguir el siguiente procedimiento: Se pesa $125 \mathrm{mg}$ de paraquat como ión en un balón de $100 \mathrm{~mL}$, se disuelve con agua desionizada, se coloca en el baño ultrasónico por 5 minutos y se lleva a volumen (solución I). Después, se toma una alícuota de $10 \mathrm{~mL}$ de la solución I y acetronitrilo, se diluye hasta $100 \mathrm{~mL}$ en un balón aforado, se filtra e introduce en el vial para hacer la medición a $290 \mathrm{~nm}$.

Cabe decir, que se preparan 10 muestras para evaluar repetibilidad y 8 muestras para valorar la precisión intermedia, en donde el coeficiente de variación debe ser mayor que el resultado de la ecuación de Horwitz al compararlos independientemente y en conjunto.

Veracidad: Se prepararon 8 muestras de igual forma que la citada anteriormente, con la diferencia de que 4 muestras de ambas metodologías se enriquecieron con $3 \mathrm{~mL}$ de la solución $B$, en caso del método de la AOAC, y $3 \mathrm{~mL}$ de la solución madre para CIPAC. El porcentaje de recuperación se debe encontrar entre 98 y $102 \%$.

Para finalizar, se analizaron 10 muestras de un mismo lote de producto comercial, según se dicta en el método de la AOAC por espectrometría UV-Vis y CIPAC por HPLC explicado previamente, para aplicar dos pruebas de hipótesis, propiamente la prueba t y el ANOVA de un factor, a los datos obtenidos para demostrar si no varían significativamente.

\section{RESULTADOS}

En la figura 1, se observan las curvas de calibración del paraquat por ambas metodologías, las cuales incluyen la ecuación de la recta y el r-cuadrado. Asimismo, para la selectividadespecificidad se realizó la comparación cualitativa de espectros de absorción y cromatogramas según método, y se demostró la idoneidad en ambos casos, al no existir interferencia alguna en cada método.

A)

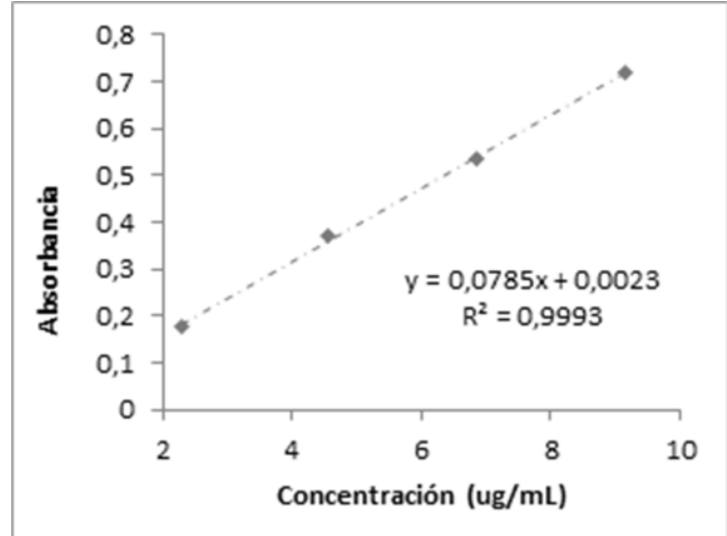

B)

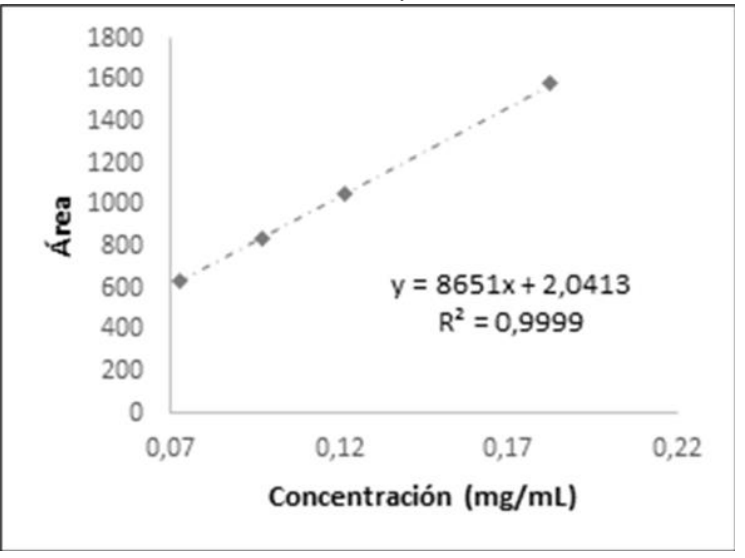

Fig. 1. Curvas de calibración de paraquat del método de la AOAC por UV (A) y del método de CIPAC por HPLC (B).

Además, en los Cuadros 1 y 2 se presentan los datos relacionados con la valoración de la veracidad, los cuales abarcan desde la concentración de la muestra inicial y enriquecida hasta el promedio del porcentaje de recuperación obtenido con ambos procedimientos analíticos. 


\section{CUADRO 1}

Evaluación de la veracidad del paraquat obtenidos con el método de la AOAC

\begin{tabular}{cccc}
\hline Muestra & $\begin{array}{c}\text { Concentración de la muestra } \\
\text { (ug/mL) } \\
\text { Inicial }\end{array}$ & $\begin{array}{c}\text { Porcentaje de } \\
\text { recuperación }\end{array}$ \\
\hline 1 & 4,6685 & 6,0371 & 100,14 \\
2 & 4,7247 & 6,1022 & 100,79 \\
3 & 4,7226 & 6,0559 & 97,55 \\
4 & 4,7440 & 6,1636 & 103,87 \\
\hline Promedio (\%) & & 100,59 \\
DSR (\%) & & 2,58 \\
\hline
\end{tabular}

\section{CUADRO 2}

Evaluación de la veracidad del paraquat obtenidos con el método de CIPAC

\begin{tabular}{|c|c|c|c|}
\hline \multirow[t]{2}{*}{ Muestra } & \multicolumn{2}{|c|}{$\begin{array}{c}\text { Concentración de la } \\
\text { muestra }(\mathrm{mg} / \mathrm{mL})\end{array}$} & \multirow{2}{*}{$\begin{array}{l}\text { Porcentaje de } \\
\text { recuperación }\end{array}$} \\
\hline & Inicial & Enriquecida & \\
\hline 1 & 0,125 & 0,163 & 103,80 \\
\hline 2 & 0,125 & 0,160 & 98,39 \\
\hline 3 & 0,124 & 0,160 & 100,43 \\
\hline 4 & 0,125 & 0,162 & 100,68 \\
\hline \multicolumn{2}{|c|}{ Promedio (\%) } & & 100,82 \\
\hline \multicolumn{2}{|l|}{ DSR (\%) } & & 2,22 \\
\hline
\end{tabular}

Asimismo, en los Cuadros 3 al 6 se expresan los valores adquiridos al evaluar la repetibilidad y precisión intermedia en ambas metodologías, los cuales comprenden desde el porcentaje masa/volumen de la muestra hasta el promedio, coeficiente de variación y ecuación de Horwitz.

\section{CUADRO 3}

Repetibilidad del paraquat en matriz con el método de la AOAC

\begin{tabular}{cccc}
\hline Muestra & $\% \mathrm{~m} / \mathbf{v}$ & Muestra & $\% \mathrm{~m} / \mathbf{v}$ \\
\hline 1 & 18,73 & 6 & 18,78 \\
2 & 18,68 & 7 & 18,72 \\
3 & 18,66 & 8 & 18,62 \\
4 & 18,76 & 9 & 18,67 \\
5 & 18,71 & 10 & 18,67 \\
\hline Promedio (\% m/v) & & 18,70 \\
CV (\%) & & & 0,26 \\
Ecuación de Horwitz & & 1,72 \\
\hline
\end{tabular}

\section{CUADRO 4}

Repetibilidad del paraquat en matriz con el método de CIPAC

\begin{tabular}{cccc}
\hline Muestra & $\% \mathrm{~m} / \mathbf{v}$ & Muestra & $\% \mathrm{~m} / \mathbf{v}$ \\
\hline 1 & 19,88 & 6 & 19,58 \\
2 & 19,21 & 7 & 19,48 \\
3 & 19,23 & 8 & 19,73 \\
4 & 19,86 & 9 & 19,86 \\
5 & 19,82 & 10 & 19,91 \\
\hline Promedio (\% m/v) & & 19,66 \\
CV (\%) & & 1,59 \\
Ecuación de Horwitz & & 1,71 \\
\hline
\end{tabular}




\section{CUADRO 5}

Precisión intermedia del paraquat en matriz con la variación del factor día con el método de la AOAC

\begin{tabular}{cccc}
\hline Muestra & $\% \mathrm{~m} / \mathbf{v}$ & Muestra & $\% \mathrm{~m} / \mathbf{v}$ \\
\hline 1 & 18,73 & 10 & 18,67 \\
2 & 18,68 & 11 & 18,76 \\
3 & 18,66 & 12 & 18,79 \\
4 & 18,76 & 13 & 18,76 \\
5 & 18,71 & 14 & 18,72 \\
6 & 18,78 & 15 & 18,81 \\
7 & 18,72 & 16 & 18,70 \\
8 & 18,62 & 17 & 18,87 \\
9 & 18,67 & 18 & 18,83 \\
\hline \multicolumn{2}{l}{ Promedio (\% m/v) } & & 18,73 \\
CV (\%) & & 0,065 \\
Ecuación de Horwitz & & 0,35 \\
\hline
\end{tabular}

\section{CUADRO 6}

Precisión intermedia del paraquat en matriz con la variación del factor día con el método de la CIPAC

\begin{tabular}{cccc}
\hline Muestra & $\% \mathbf{~ m / v}$ & Muestra & $\% \mathbf{~ m / v}$ \\
\hline 1 & 19,88 & 10 & 19,91 \\
2 & 19,21 & 11 & 19,70 \\
3 & 19,23 & 12 & 19,87 \\
4 & 19,86 & 13 & 19,72 \\
5 & 19,82 & 14 & 19,71 \\
6 & 19,58 & 15 & 20,08 \\
7 & 19,48 & 16 & 20,08 \\
8 & 19,73 & 17 & 19,94 \\
9 & 19,86 & 18 & 20,01 \\
\hline \multicolumn{2}{l}{ Promedio (\% m/v) } & & 19,76 \\
CV (\%) & & & 0,25 \\
Ecuación de Horwitz & & 1,27 \\
\hline
\end{tabular}

Por otra parte, en el Cuadro 7 a continuación se puede observar los resultados de la comparación entre los dos métodos, el cual incluye el porcentaje masa/volumen, el promedio, la desviación estándar, el coeficiente de variación y los valores de la T experimental y teórica.

Finalmente, en la figura 2 se puede ver la gráfica de valores individuales en la comparación de ambos procedimientos analíticos.

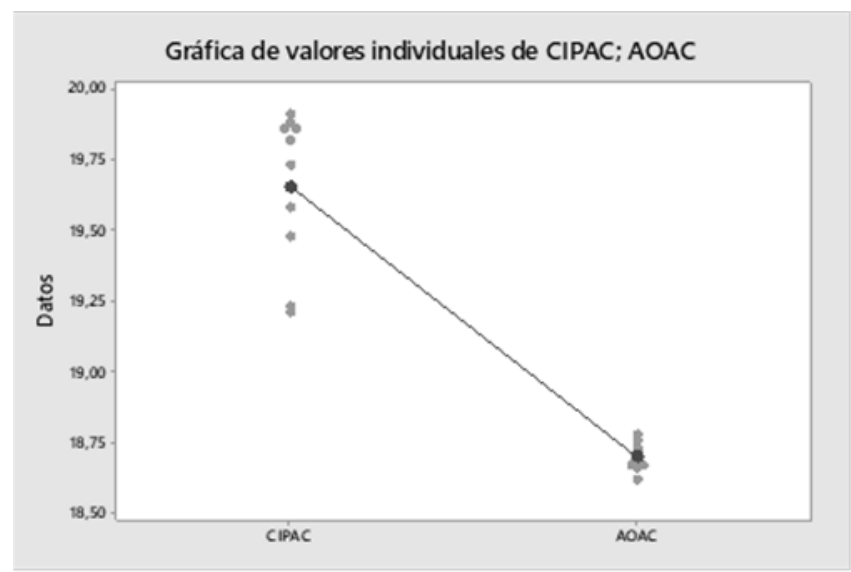

Fig. 2. Gráfica de valores individuales del ANOVA de un factor al comparar ambas metodologías 


\section{CUADRO 7}

Resultados de la Prueba T de dos colas de un producto comercial para la comparación de ambos métodos

\begin{tabular}{lccc}
\hline \multirow{2}{*}{ Muestra } & \multicolumn{2}{c}{$\% \mathrm{~m} / \mathrm{v}$} \\
& AOAC & CIPAC \\
\hline 2 & 18,73 & 19,88 \\
& 1 & 18,68 & 19,21 \\
4 & 18,66 & 19,23 \\
& 5 & 18,76 & 19,86 \\
& 6 & 18,71 & 19,82 \\
& 7 & 18,78 & 19,58 \\
& 8 & 18,72 & 19,48 \\
& 9 & 18,62 & 19,73 \\
& 10 & 18,67 & 19,86 \\
Promedio (\% m/v) & 18,67 & 19,91 \\
Desviación estándar (\% m/v) & 18,70 & 19,66 \\
CV (\%) & 0,049 & 0,27 \\
T experimental & 0,26 & 1,36 \\
T teórica & \multicolumn{2}{c}{11,09} \\
\hline \multicolumn{2}{l}{1,96} \\
\end{tabular}

\section{DISCUSIÓN}

En una validación se debe evaluar el intervalo de trabajo, en donde el laboratorio considere tanto la linealidad del método como el procedimiento de calibración propuesto, sin embargo, en una verificación se evalúa solamente el intervalo de trabajo del instrumento, ya que el del método fue corroborado durante el desarrollo del mismo. Así pues, al valorar la linealidad del equipo se debe demostrar, al menos, sobre el rango de $\pm 20 \%$ de la concentración del analito y graficar las señales, en las cuales se espacien uniformemente las concentraciones seleccionadas en el rango. Inicialmente, al momento de analizar los datos se realiza una inspección visual de la curva de calibración, luego se confirma la relación entre la concentración y la respuesta del instrumento revisando las estadísticas de regresión, y por último el analista puede decidir si el procedimiento de calibración propuesto indicado en el método es apropiado, es decir, si con la investigación se efectúa aportación de evidencia objetiva de que un elemento dado satisface los requisitos especificados (Eurolab, 2016). Por ende, en el Laboratorio de Control de Calidad de Agroquímicos se realizó solamente una curva de calibración para cada metodología en un rango de $\pm 20 \%$ de la concentración de paraquat, ya que según se muestra en la figura 1 , ambos procedimientos analíticos poseen un r-cuadrado mayor a 0,99, el cual es el criterio de aceptación (CIPAC, 2003), de modo que, se puede decir que tanto el método de CIPAC como el de la AOAC dan respuesta lineal en los equipos en los cuales se realizó la verificación.

Asimismo, la señal cualitativa obtenida es solamente del paraquat tanto con el equipo HPLC como por el espectrofotómetro UV-Vis, ya que cuando se analiza el blanco no se detecta ninguna absorción, mientras que al cuantificar las muestras o patrones existe una señal, es decir, se declara que ambos métodos son específicos y selectivos.

También según CIPAC (2003), los plaguicidas que tienen una concentración de ingrediente activo mayor al $10 \%$ deben adquirir un porcentaje de recuperación entre $98 \%$ a $102 \%$. En los cuadros 1 y 2 se pueden ver los porcentajes de recuperación tanto del procedimiento analítico de la AOAC como de CIPAC, siendo los mismos $100 \%$ y $101 \%$ respectivamente, es decir, ambas metodologías se consideran veraces al encontrarse dentro del intervalo de aceptación. Además, los desvíos relativos 
promedios son menores a 3\%, lo que expresa que hay una buena precisión de los resultados (Ministerio de Salud, s.f.).

Igualmente, en los cuadros 3, 4, 5 y 6 se puede notar los resultados de la evaluación de la repetibilidad y precisión intermedia, donde el coeficiente de variación en todos ellos es menor a la ecuación de Horwitz y a $3 \%$, significando que la concentración de paraquat va a ser la misma aunque se cambie el factor día, a razón de lo cual se consideran los datos repetibles y con una adecuada precisión (CIPAC, 2003).

Por otro lado, según el decreto N²7035-MAG-MEIC (1998) los plaguicidas que poseen un porcentaje de ingrediente activo entre $10 \%$ y $25 \%$ pueden variar en un $\pm 6 \%$ de lo establecido en la etiqueta. Así pues, al analizar los resultados alcanzados del producto comercial en el cuadro 7 , se determinó que por el método de la AOAC se obtiene un promedio de $18,73 \% \mathrm{~m} / \mathrm{v}$, por lo cual, ante el valor declarado en la etiqueta de $20 \% \mathrm{~m} / \mathrm{v}$, se presenta un subetiquetado de un $1,27 \%$. Además al compararlo con el decreto previamente mencionado, se puede ver que por medio de este procedimiento analítico los resultados obtenidos cumplen con la legislación del país.

Por su parte al analizar los datos que se recabaron según el método de la CIPAC, se puede apreciar que el promedio es un $19,76 \% \mathrm{~m} / \mathrm{v}$, definiéndose un subetiquetado del ingrediente activo de un $0,24 \%$, por lo que se observa que es un resultado muy cercano al valor teórico de un $20 \% \mathrm{~m} / \mathrm{v}$ y que también a su vez cumple con lo establecido en la legislación costarricense para este tipo de producto.

Ahora bien, al comparar los datos obtenidos entre ambas metodologías por medio de la prueba $\mathrm{T}$ de dos colas, se determina que los resultados varían entre sí, ya que el valor de $T$ experimental, específicamente 11,09 es mayor que el de T teórico, siendo este 1,96; o sea, se rechaza la hipótesis nula, la cual es: que los resultados obtenidos por los dos procedimientos analíticos son estadísticamente iguales. Lo anterior se confirma al realizar el ANOVA de un factor, debido a que el valor $\mathrm{p}$ es menor al nivel de significancia, siendo estos 0,00 y 0,05 respectivamente, lo que indica que la concentración de paraquat adquirida por las dos metodologías varía significativamente. Dicha diferencia se puede adjudicar a la dispersión de los datos por medio del procedimiento analítico de CIPAC, tal como se ve en la figura 2, a pesar de que previamente se declara que los datos obtenidos por medio del HPLC tienen una buena precisión.

Cabe mencionar que este resultado es válido para la comparación de los dos métodos con un solo analista, por tanto, se debe plantear si el mismo fenómeno ocurre cuando se realiza el mismo ejercicio con otros analistas o con el conjunto de datos de cada método a largo plazo con distintos analistas. Esto debido a que la variación del método de la AOAC presenta un coeficiente de variación anormalmente bajo de apenas un 15\% del valor de referencia de Horwitz.

\section{AGRADECIMIENTOS}

Agradecemos al personal del Laboratorio de Control de Calidad de Agroquímicos, del Servicio Fitosanitario del Estado en Costa Rica, por la apertura para realizar trabajos como este, y por todo el apoyo brindado durante el proceso de indagación.

\section{ÉTICA, CONFLICTO DE INTERESES Y DECLARACIÓN DE FINANCIAMIENTO}

Los autores declaran haber cumplido con todos los requisitos éticos y legales pertinentes, tanto durante el estudio como en el manuscrito; que no hay conflictos de interés de ningún tipo, y que todas las fuentes financieras se detallan plena y claramente en la sección de agradecimientos. Asimismo, están de acuerdo con la versión editada final del documento. El respectivo documento legal firmado se encuentra en los archivos de la revista. 
La contribución de los autores es como se detalla a continuación: E.B.B.: Desarrollo experimental del trabajo, búsqueda de literatura y análisis de resultados. E.P.L.: Guía en lo académico del desarrollo del trabajo investigativo, aporte en criterios de validación-comparación de metodologías, orientación en apartados del informe de la investigación que daría paso al artículo y montaje del artículo científico según formato. R.V.L.: Guía experimental en la investigación, aporte de criterios para validar y comparar las metodologías y revisión del manuscrito"

\section{REFERENCIAS}

Agencia de Protección Ambiental de Estado Unidos. (2019). Dicloruro de Paraquat. Recuperado de: https://espanol.epa.gov/seguridad-laboral-al-usar-pesticidas/dicloruro-de-paraquat

Agrologica. (2012). Los plaguicidas: presentación, abreviaturas y orden de mezcla. Recuperado de: http://blog.agrologica.es/los-plaguicidas-presentacion-abreviaturas-y-orden-de-mezcla/

Alza-Camacho, W. R., García-Colmenares, J. M., \& Chaparro-Acuña, S. P. (2016). Determinación voltamétrica de paraquat y glifosato en aguas superficiales. Ciencia y Tecnología Agropecuaria, 17(3), 331-345. Recuperado de: https://www.redalyc.org/articulo.oa?id=4499/449946663002

Araya, J. (03 de junio 2015). Costa Rica es el consumidor más voraz de plaguicidas en el mundo. Seminario Universidad. Recuperado de: https://ojoalclima.com/costa-rica-es-el-consumidor-mas-voraz-de-plaguicidas-en-elmundo/\#: :text=Costa\%20Rica\%20es\%20el\%20consumidor\%20m\%C3\%A1s\%20voraz\%20de\%20plaguicidas\%20e n\%20el\%20mundo,-

por\%20Jorge\%20Araya\&text=Comparado\%20con\%20otros\%20pa\%C3\%ADses\%2C\%20Costa,agr\%C3\%B3nomo\% 20e\%20investigador\%20del\%20IRET.

CIPAC. (2003). Guidelines on method validation to be performed in support of analytical methods for agrochemical formulations. No. 3807. Recuperado de: https://www.cipac.org/images/pdf/validat.pdf

Decreto N²7035-MAG-MEIC. (1998). Norma RTCR 171: 1991 Plaguicidas y coadyuvantes. Tolerancias permitidas para la concentración del ingrediente activo. Diario oficial la Gaceta No. 174, Costa Rica. Recuperado de: http://reventazon.meic.go.cr/informacion/gaceta/1998/setiembre/27035.pdf

Delgado, J.J. (2015). Validación y aplicación de un método de análisis para la determinación de terpiridinas totales en materia prima de Dicloruro de Paraquat por medio de cromatografía de gases-masas (Tesis de Licenciatura), Universidad de Costa Rica San José, Costa Rica. Recuperado de: http://repositorio.sibdi.ucr.ac.cr:8080/jspui/handle/123456789/3304?mode=full\&submit_simple=Mostrar+el+re gistro+Dublin+Core+completo+del+\%C3\%ADtem+

Dierksmeier, G., Hernández, R., Ricardo, C., Linares, C., García, M., Suárez, B., Orta, L., \& Lazo, A. (2002). Contaminación por plaguicidas en la Ciénaga de Zapata y su Zona Costera. Fitosanidad, 6(2), 27-33. Recuperado de: http://www.redalyc.org/articulo.oa?id=209118291006

Echemendía, M. (s.f.). El manejo de plagas y el uso de plaguicidas en la agricultura. Recuperado de: https://www.monografias.com/trabajos98/manejo-plagas-y-uso-plaguicidas-agricultura/manejo-plagas-y-usoplaguicidas-agricultura.shtml

Eurolab España. P.P. Morillas y colaboradores. (2016). Guía Eurachem: La adecuación al uso de los métodos analíticos Una Guía de laboratorio para la validación de métodos y temas relacionados.

Inquisa. (s.f.). Hoja de datos de seguridad ERASER 20 SL. Recuperado de: http://www.agroproca.com/productos/documentacion/msds/Eraser\%2020\%20SL.pdf

Luna, J. R., Di Bernardo, M. L., García, M. Y., Ovalles, F., \& Calderón, L. (2008). Determinación de paraquat en orina utilizando un sistema de inyección en flujo continuo. Acta Bioquímica Clínica Latinoamericana, 42(2), 251-259. Recuperado de: http://www.redalyc.org/articulo.oa?id=53542211 
Marín, R. (2003). Fisicoquímica y microbiología de los medios acuáticos. Tratamiento y control de calidad de aguas. Cartagena, España: Ediciones Díaz de Santos. Recuperado de https://books.google.co.cr/books/about/Fisicoqu\%C3\%ADmica_y_microbiolog\%C3\%ADa_de_los_m.html?id=5S WUswEACAAJ\&redir_esc=y

Mendoza, A. (2011). Información general sobre los herbicidas. Dirección general de investigación sobre la contaminación urbana y regional. Recuperado de: https://studylib.es/doc/5789457/informaci\%C3\%B3n-general-sobre-losherbicidas

Ministerio de Salud. (s.f.). Guía de validación de métodos analíticos. Recuperado de: https://www.ministeriodesalud.go.cr/index.php/biblioteca-de-archivos/tramites/registro-de-productos-deinteres-sanitario/medicamentos-1/documentos-de-interes-3/guias-de-registro-medicamentos/2472-guia-devalidacion-de-metodos-analiticos/file

Moctezuma, E., Zamarripa, H., \& Leyva, E. (2003). Degradación fotocatalítica de soluciones de alta concentración de paraquat. Revista Internacional de Contaminación Ambiental, 19(3), 117-125. Recuperado de: http://www.redalyc.org/articulo.oa?id=37019302

Molina, Y., Flores, M., Balza, A., Benítez, P., \& Miranda, L. (2012). Niveles de plaguicidas en aguas superficiales de una región agrícola del Estado Mérida, Venezuela, entre 2008 y 2010. Revista Internacional de Contaminación Ambiental, 28(4),289-331.Recuperado de: http://www.redalyc.org/articulo.oa?id=37025139010

Morales, R. (2013). Metodología de análisis del riesgo por contaminación de agroquímicos: cuenca del Río San Blas, Costa Rica. Revista Costarricense de Salud Pública, 22(1), 35-44. Recuperado de: https://www.scielo.sa.cr/pdf/rcsp/v22n1/art07v22n1.pdf

Reynoso, P., \& Carvalho, R. J. (2010). Degradación del pesticida Paraquat a través del proceso de ozonización. Revista CENIC, 41, 1-11. Recuperado de: http://www.redalyc.org/articulo.oa?id=181620500043

Reyes, J., Vásquez, R., \& González, T. (2010). Introducción a la agroquímica. México, Editorial BUAP. Recuperado de: https://books.google.co.cr/books/about/Introducción_a_la_Agroquímica.html?id=DIUWWgEZr5YC\&redir_esc=y

Salazar, N., \& Aldana, M. (2011). Herbicida Glifosato: Usos, toxicidad y regulación. Revista Ciencias Biológicas y de la Salud, 13(2), 23-28. DOI: https://doi.org/10.18633/bt.v13i2.83 